\title{
Article
}

\section{Enhanced selectivity of hydrogel-based molecularly imprinted polymers (HydroMIPs) following buffer conditioning.}

El-Sharif, HF, Phan, QT and Reddy, Subrayal M

Available at http://clok.uclan.ac.uk/13671/

El-Sharif, HF, Phan, QT and Reddy, Subrayal M ORCID: 0000-0002-7362-184X (2014) Enhanced selectivity of hydrogel-based molecularly imprinted polymers (HydroMIPs) following buffer conditioning. Anal Chim Acta, 809.155 $-161$.

It is advisable to refer to the publisher's version if you intend to cite from the work.

For more information about UCLan's research in this area go to

http://www.uclan.ac.uk/researchgroups/ and search for <name of research Group>.

For information about Research generally at UCLan please go to http://www.uclan.ac.uk/research/

All outputs in CLoK are protected by Intellectual Property Rights law, including Copyright law. Copyright, IPR and Moral Rights for the works on this site are retained by the individual authors and/or other copyright owners. Terms and conditions for use of this material are defined in the policies page. 


\section{Enhanced selectivity of hydrogel-based molecularly imprinted polymers (HydroMIPs)}

\section{2 following buffer conditioning.}

4 Hazim F. EL-Sharif, Quan T. Phan, Subrayal M. Reddy*

5 Department of Chemistry, Faculty of Engineering and Physical Sciences, University of 6 Surrey, Guildford, Surrey, GU2 7XH, UK

7

\section{*Corresponding Author}

9 Tel : +44 (0) 1483686396, s.reddy@surrey.ac.uk

\section{Abstract}

12 We have investigated the effect of buffer solution composition and $\mathrm{pH}$ during the preparation,

13 washing and re-loading phases within a family of acrylamide-based molecularly imprinted

14 polymers (MIPs) for bovine haemoglobin (BHb), equine myoglobin (EMb) and bovine

15 catalyse (BCat). We investigated water, phosphate buffer saline (PBS), tris(hydroxymethyl)aminomethane (Tris) buffer and succinate buffer. Throughout the study

17 MIP selectivity was highest for acrylamide, followed by N-hydroxymethylacrylamide, and then N-iso-propylacrylamide MIPs. The selectivity of the MIPs when compared with the

19 NIPs decreased depending on the buffer conditions and $\mathrm{pH}$ in the order of Tris $>$ PBS >

20 succinate. The Tris buffer provided optimum imprinting conditions at $50 \mathrm{mM}$ and $\mathrm{pH} 7.4$, and MIP selectivities for the imprinting of BHb in polyacrylamide increased from an initial $8: 1$ to a $128: 1$ ratio. It was noted that the buffer conditions for the re-loading stage was important for determining MIP selectivity and the buffer conditions for the preparation stage

24 was found to be less critical. We demonstrated that once MIPs are conditioned using Tris or

25 PBS buffers ( $\mathrm{pH} 7.4)$ protein reloading in water should be avoided as negative effects on the 
26 MIP's imprinting capability results in low selectivities of 0.8:1. Furthermore, acidifying the

$27 \mathrm{pH}$ of the buffer solution below pH 5.9 also has a negative impact on MIP selectivity

28 especially for proteins with high isoelectric points. These buffer conditioning effects have

29 also been successfully demonstrated in terms of MIP efficiency in real biological samples,

30 namely plasma and serum.

31

32 Keywords: Molecular imprinting; Protein binding; Selectivity; Biomimetic material; pH;

33 Biocompatibility

34 


\section{Introduction}

Molecularly imprinted polymers (MIPs) are polymers which have been synthesized to have tailor-made selectivity for a template molecule. Hydrogels are insoluble, crosslinked polymer network structures composed of hydrophilic homo- or hetero-co-polymers, which have the ability to absorb significant amounts of water [1]. Molecular imprinting using hydrogels (HydroMIPs) have been well documented, with a vast array of monomers currently being used for molecular imprinting [2]. Monomers that have commonly been used for noncovalent molecular imprinted hydrogels are generally chosen on their ability to form weak hydrogen bonds between the monomer and the template [1, 3, 4]. Acrylamide-based hydrogels are known to be very inert, offer hydrogen bonding capabilities, and are biocompatible [5]. For these reasons, acrylamide has been commonly used for molecular imprinting [6-10].

Historically, molecular imprinting has been used for low molecular weight non-biological molecules such as drugs and pesticides $[11,12]$. This has been due in part to the fact that MIPs have been hard to adapt to aqueous conditions due to the specific polar interactions between good imprinted sites and the analyte which become weakened in the presence of a polar solvent, and to the non-specific (hydrophobic) interactions between other small molecules and the gel which become strengthened [6]. However, popularity for imprinting for

54 large macromolecule templates such as proteins has increased in the past decade, with a view to developing sensors and new diagnostics for disease markers [13]. Organic solvents traditionally employed in creating MIPs are not well suited to imprinting biological molecules such as proteins, as the non-polar side chains are more soluble the hydrophobic interactions that maintain the highly folded quaternary structure are weakened considerably. 
6]. Also, for bioanalytical applications, it is desirable for the resultant MIPs to be efficiently used under aqueous conditions [11]. Work carried out by Andersson [11, 12] suggested that upon changing from organic solvent to aqueous based incubation, the selectivity of the MIPs can be changed. It was suggested that in organic solvents the imprints recognise subtle differences in polar functionalities of the template molecule, and in aqueous media the hydrophobic parts of the template molecule are more efficiently recognised [11].

66

67 One of the holy grails for the molecular imprinting community is to achieve binding affinities for MIPs that can be comparable to the high selectivity offered for instance antibody-antigen binding [13]. Another example, the biotin-avidin interaction, is renowned for having a large binding constant of $10^{15} \mathrm{M}^{-1}$ [13]. This interaction is purely non-covalent, but the strength of interaction comes from 15 amino acid residues on the avidin being in optimum positions to specifically interact with the vitamin, biotin; approximately half through hydrogen bonding interactions and the other half of the residues through hydrophobic interactions [13]. The high specificity is compounded by the flexibility of the protein to subtly change its conformation in order to lock into place upon biotin binding. This is quite a complex series of events which is made to look easy by such natural systems. MIPs are typically highly cross-linked systems and by virtue of their rigid structure are therefore unable to offer many degrees of freedom to allow similar capture and locking to take place. However, hydrogel-based MIPs are able to swell and contract depending on solvent, ionic strength and the presence of other dissolved components in solution $[1,3,11,14]$. If these parameters can be optimised to improve selective binding within MIPs compared to non-imprinted polymer controls, we could go some way in improving the reputation of MIPs as biomimetic and antibody-like materials [14]. 
84 When protein imprinting, one often overlooked parameter is protein stability [10-12].

85 Conventionally, protein stability is achieved using lyophilisation, freezing and homogenization techniques [15]. However in a MIP system, buffers offer a more attractive choice for sustaining biological molecules in their native state, and thus have been extensively used in a range of chemical and biochemical assays. In some cases, the assays make use of a solid support upon which a biorecognition molecule (such as an enzyme or antibody) is immobilised. There have been recent studies that have focused on the effect of surface modifications and protein modifications on biomolecule stability $[15,16]$. For example, Wei [15] demonstrated that buffer type as well as buffer concentration can have significant effects on protein adsorption onto surfaces. It was suggested that at $\mathrm{pH} 7.4$, protein adsorption increased monotonically with a Tris buffer, while a PBS buffer induced negative adsorption effects. This was attributed to the possibility of various phosphate ions competing to adsorb with protein molecules [15].

This paper aims to investigate the effects that aqueous buffer composition has on the specific rebinding and/or non-specific adsorption of template protein molecules into hydrogel-based MIPs compared with control non-imprinted polymers (NIPs). We will demonstrate that MIP surfaces can also be affected (e.g. positively in terms of their binding affinity for target protein) depending on the chemical nature of the buffer and its $\mathrm{pH}$.

103

\section{Experimental}

\subsection{Materials and reagents}

106 Acrylamide (AA), N-hydroxymethylacrylamide (NHMA), N-iso-propylacrylamide (NiPAm),

107 N,N-methylenebisacrylamide (bis-AA), ammonium persulphate (APS), N,N,N,N-

108 tetramethylethyldiamine (TEMED), sodium dodecyl-sulphate (SDS), glacial acetic acid 
109 (AcOH), tris(hydroxymethyl)-amine (Tris-base), tris(hydroxymethyl)-amine hydrochloride

110 (Tris-HCl), phosphate buffered saline (PBS) tablets (137 mM NaCl; $27 \mathrm{mM} \mathrm{KCl} ; 10 \mathrm{mM}$

$\left.111 \mathrm{Na}_{2} \mathrm{HPO}_{4} ; 1.76 \mathrm{mM} \mathrm{KH} \mathrm{PO}_{4}\right)$, succinic acid, bovine haemoglobin (BHb), bovine liver

112 catalase (BCat), and equine heart myoglobin (EMb) were all purchased from Sigma-Aldrich,

113 Poole, Dorset, UK. Sieves $(75 \mu \mathrm{m})$ were purchased from Endecotts Ltd. and Inoxia Ltd., UK.

114 Pooled plasma and serum samples from human volunteers were used in the biocompatibility

115 studies.

116

\section{$117 \quad 2.2$ Spectrophotometric analysis}

118 Calibration curves in MilliQ water, buffer solutions, and 10\% AcOH:SDS were prepared for

$119 \mathrm{BHb}, \mathrm{BCat}$ and EMb. Spectral scans revealed peak wavelengths for BHb in MilliQ water and

$12010 \% \mathrm{AcOH}: \mathrm{SDS}$ to be $405 \mathrm{~nm}$ and $395 \mathrm{~nm}$ respectively. All buffer solutions for BHb

121 exhibited a peak wavelength at $406 \mathrm{~nm}$, with the exception of succinate buffer $\mathrm{pH} 2.9$, which

122 exhibited a peak wavelength at $367 \mathrm{~nm}$. Peak wavelengths for BCat in MilliQ water, Tris

123 buffer and 10\% AcOH:SDS were found to be $405 \mathrm{~nm}, 404 \mathrm{~nm}$ and $392 \mathrm{~nm}$ respectively. Peak

124 wavelengths for EMb in MilliQ water, Tris buffer and 10\% AcOH:SDS were found to be 410

$125 \mathrm{~nm}, 408 \mathrm{~nm}$ and $396 \mathrm{~nm}$ respectively. Analysis and subsequent determination of protein

126 concentration in appropriate media was performed at specific peak wavelengths using a UV

127 mini-1240 CE spectrophotometer (Shimadzu Europa, Milton Keynes, UK).

128

$129 \quad 2.3$ Hydrogel production method

130 Hydrogel MIPs were synthesised by separately dissolving AA (54 mg), NHMA (77 mg),

131 NiPAm $(85.6 \mathrm{mg})$ and bis-AA as cross-linker $(6 \mathrm{mg}),(8.5 \mathrm{mg})$ and $(9.5 \mathrm{mg})$ respectively

132 along with template protein $(12 \mathrm{mg})$ in $1 \mathrm{~mL}$ of either PBS or MilliQ water. The solutions

133 were purged with nitrogen for 5 minutes, followed by an addition of $20 \mu \mathrm{L}$ of a $10 \%(w / v)$ 
134 APS solution and $20 \mu \mathrm{L}$ of a 5\% (v/v) TEMED solution. Polymerisation occurred overnight at room temperature giving final crosslinking densities of $10 \%$. MilliQ water is used as a hydrogel preparation standard, except when investigating the effect of changing the gel

137 preparation conditions and optimising template rebinding using PBS. For every MIP created

138 a non-imprinted control polymer (NIP) was prepared in an identical manner but in the 139 absence of protein.

\subsection{Hydrogel conditioning}

142 After polymerization, the gels were granulated separately using a $75 \mu \mathrm{m}$ sieve. Of the

143 resulting gels, $0.5 \mathrm{~mL}$ were transferred into $1.5 \mathrm{~mL}$ centrifuge eppendorf tubes and

144 conditioned by washing with five $1 \mathrm{~mL}$ volumes of either MilliQ water or buffer solution (50 mM Tris $\mathrm{pH}$ 7.4; $50 \mathrm{mM}$ succinate $\mathrm{pH}$ 7.4; 50 mM succinate $\mathrm{pH}$ 2.9; $150 \mathrm{mM}$ PBS pH 7.4; and PBS pH 4.7). This was followed by five $1 \mathrm{~mL}$ volumes of a $10 \% \mathrm{AcOH}$ :SDS eluent (pH 2.8). A further five $1 \mathrm{~mL}$ washes of either MilliQ water or buffer solution were conducted to remove any residual AcOH:SDS eluent and equilibrate the gels. The PBS and succinate buffer solutions used in this study were adjusted to $\mathrm{pH} 4.7$ and $\mathrm{pH} 7.4$ using $1 \mathrm{M} \mathrm{HCl}$ and 1 $\mathrm{M} \mathrm{NaOH}$ respectively. Each conditioning step was followed by a centrifugation using an eppendorf mini-spin plus centrifuge for 3 minutes at $6000 \mathrm{rpm}$ (RCF: 2419 x g). All supernatants were collected for analysis by spectrophotometry.

\subsection{Rebinding optimisations}

155 Once the gels $(0.5 \mathrm{~mL})$ were equilibrated, a $1 \mathrm{~mL}$ protein solution 'load' prepared in either MilliQ water or buffer solution, containing $3 \mathrm{mg}$ of protein was added to the target MIPs and NIP controls. The MIPs and NIPs were then washed with four $1 \mathrm{~mL}$ volumes of either MilliQ water or buffer solution 'wash', followed by five $1 \mathrm{~mL}$ washes of $10 \%$ AcOH:SDS 'elute'. 
159 Each step (load, wash, and elute) was followed by a centrifugation as previously described,

160 and again all supernatants were collected for analysis by spectrophotometry.

\section{3. Results and discussion}

\section{$163 \quad 3.1$ Rebinding Optimisations}

164 The molecular imprinting effect or imprinting efficiency throughout this work was characterised by the rebinding capacity exhibited by the protein-specific MIP in relation to the control NIP. This can be represented as a selectivity ratio ( $\alpha)$ using Eq. (1).

$$
\alpha=\frac{[\text { specific binding }]_{M I P}}{[\text { specific binding }]_{N I P}}
$$

170 Using this equation, the selectivity ratio, $\alpha$, is generally determined using the specific-bound

171 protein concentrations recovered in the 10\% SDS:AcOH supernatant 'elute' fractions. This

172 heavily relies on the $10 \%$ SDS:AcOH being $100 \%$ efficient in removing/eluting protein;

173 however only a 90\% efficiency has been reported previously [6]. Therefore, in order to evaluate precisely how much protein is retained by the MIP, an alternative approach is to calculate the specific-bound protein by subtracting the recovered non-specific protein in the 'load' and 'wash' fractions from the initial protein additions. Table 1 illustrates the experimental data in terms of selectivity ratios $(\alpha)$ calculated using the latter method.

178 Interestingly, the resulting MIP selectivity shows a varying affinity for different proteinpolymer combinations depending on the hydrophobicity of the polymer. It can be seen that MIPs based on polyacrylamide (polyAA) showed the most promising results in terms of imprinting efficiency closely followed by poly N-hydroxymethylacrylamide (polyNHMA),

182 then poly $\mathrm{N}$-iso-propylacrylamide (polyNiPAm). 
184 Fig.1 illustrates the quantified imprinting effect for BHb in polyAA, polyNHMA and polyNiPAm hydrogel MIPs. Distinctive MIP and NIP loading characteristics are seen in the differing degrees of template $\mathrm{BHb}$ protein recovered in supernatant phases after protein loading in water (Load), water washing (Wash) and 10\% SDS:AcOH elution (Elute) phases.

188 It is evident that the template $\mathrm{BHb}$ is easily recognised and bound by recognition sites in the 189 specific cavity-based MIPs from the negligible amounts of unbound BHb observed in the 190 Load supernatant, while the Elute fractions comprise high concentrations of the specifically 191 rebound protein. This strongly suggests that the template BHb has been specifically bound 192 within MIP specific cavities. Furthermore, the total amount of protein recovered in the $193 \mathrm{MIP}_{\text {polyAA }}(1.5 \mathrm{mg})$ after rebinding and elution with $10 \% \mathrm{SDS}: \mathrm{AcOH}$ is less than the amount 194 used for rebinding ( $3 \mathrm{mg}$ ), suggests some irreversible rebinding of protein to the MIP. The 195 NIPs however, remain unselective and essentially reject the protein as they lack imprinted cavities and only allow for non-specific adsorption of protein.

198 It should be noted that for all MIPs approximately $50 \%$ of the imprinted template was

199 recovered and quantified using spectrophotometric analysis during the initial conditioning washes (MilliQ water and/or buffer, and the $10 \%$ ratio (w/v) of SDS:AcOH). The time allowed for template removal is specified within the washing procedures, and the last wash fractions were not observed to contain any protein. Therefore we are confident that the remaining $50 \%$ of the template protein did not continue to leach out during rebinding studies.

204 An issue to address is that the diffusivity of proteins in cross-linked polymer matrices is 205 rather slow when looking at surface binding [16-17]. Generally polymer geometry, polymer hydration, cross-linker density, protein size and temperature all play a role in the time needed

207 for a protein to diffuse into the polymer matrix and to reach equilibrium [17]. For instance, 208 the molecular weight of BHb is approximately four times higher than that of EMb [16-17]. 
209 Thus the difference in size leads to a retarded movement of BHb in the cross-linked polymer

210 matrix and therefore more time is expected to be required in order to reach equilibrium.

211 However, MIPs exhibited here are ground down (75um) and exhibit a bulk gel effect.

212 Therefore the required incubation time was validated using polyAA-gels before affinity was

213 properly assessed. It should be noted that equilibrium of $\mathrm{BHb}$ and $\mathrm{EMb}$ upon incubation with

214 their corresponding MIPs was reached at different times. However after 20 min overall

215 loading time, concentrations for $\mathrm{BHb}$ in the supernatant did not continue to decrease,

216 suggesting that equilibrium binding had been reached. Therefore an optimised time (20 min)

217 was applied for protein equilibrium binding.

219 In order to alter MIP binding selectivity, buffer solutions were incorporated, and specifically

220 chosen as they are used in biological (antibody-based) assays, to stabilise proteins. Their application in conjunction with MIPs that could potentially replace antibodies was therefore of value if MIP selectivity can be improved as a function of stabilising the protein conformation by judicious choice of buffer medium. Table 2 lists selectivity ratios $(\alpha)$ for the $\mathrm{BHb}-\mathrm{MIP}_{\text {polyAA }}$ depending on the various buffer and $\mathrm{pH}$ variation for MIP preparation, conditioning series (washing water/buffer), and BHb protein loading. Experiments 1 to 10 summarise the effect of changing solution conditions between water and PBS and the resultant selectivity is given in decreasing order. Initially the presence of PBS is beneficial; however a comparison of experiments 1 and 6, despite identical preparation and conditioning, shows a 27:1 and a 7:1 selectivity respectively. This is due to dilution of the PBS ionic strength within the MIP when loading in water and suggests that the loading stage is more crucial in affecting selectivity than either the preparation or the conditioning stages. This shift

232 is possibly causing an expansion in the gel during the loading phase due to the simultaneous 233 movement of buffer ions out of the gel, and water and protein into the gel. The movement of 
water into a gel (by osmosis) to dilute the ionic strength within the gel is a well-documented phenomenon [18]. The osmotic pressure of the gel is a function of the salt present within the gel and varies with the nature of the salt in the order of the lyotropic series. The lyotropic series is a classification of ions in order of their ability to salt out (dissolve) or salt-in (precipitate) proteins and hydrogel polymers. While early members of the series such as phosphate decrease the solubility of non-polar molecules and cause salting out, later members in the series increase the solubility of non-polar molecules (salting-in) the driving force for water diffusion into the gel is the concentration gradient of phosphate ions within the gel and outside the gel. It is likely that during this state of dynamic macro and nano-structural change in the MIP due to ingress of water, the protein although in the native state is unable to significantly bind with the selective cavities in the MIP [19]. The ionic strength impact of the PBS on MIP selectivity is further confirmed when using diluted PBS (experiments 7 and 8) and only PBS in MIP preparation (experiments 5 and 10). However, when we investigated reloading in sodium chloride solution only (no phosphate salts), the selectivity ratio was 1:1. Therefore, it would appear that the phosphate buffer salts are contributing to the improved selectivity by retaining the $\mathrm{pH}$ at 7.4 and providing a more stable protein environment.

Experiments 11 and 12 show the effect of using a Tris buffer at $\mathrm{pH} 7.4$ for the conditioning and loading phases, the only difference being the medium used for initial preparation of the MIP. Interestingly the MIP prepared in water gives a much elevated selectivity ratio of 128:1 compared with MIP prepared in PBS (30:1 selectivity). Both are superior to the other conditions studied and it would appear that conditioning and loading with Tris is having a beneficial effect on the selective binding of BHb to the MIP compared with the NIP. It is possible that although the $\mathrm{pH}$ is constant at 7.4 the initial PBS ions embedded within the MIP 
matrix. A similar effect can also be seen when comparing experiments 1 and 3, where PBS

260 yields a higher selectivity when initially preparing the MIP in water rather than in PBS. A

261 comparison of the buffers' structures in Table 3 illustrates the three undissociated hydroxy

262 groups present in the Tris buffer $(\mathrm{pKa}=8.1)$. It is plausible that the latter are able to

263 hydrogen bond with both MIP and template $\mathrm{BHb}$ protein. This in turn aids in stabilising the

264 native protein structure in the fine protein-selective cavity within the MIP and provides ideal

265 conditions for the protein to selectively bind with the MIP at an optimum $\mathrm{pH}$ of 7.4. This

266 hypothesis is validate by control Tris buffer experiments 13 and 14 at lower (5.4) and higher

267 (9.4) $\mathrm{pH}$ values which both exhibit lower selectivity values of 60:1.

269 Experiments 15-18 illustrate the presence of succinate buffer $(\mathrm{pKa}=4.2)$, and at either high

270 (7.4) or low (2.9) pH MIP selectivity is low. However, a lower selectivity is exhibited for succinate at $\mathrm{pH}$ 2.9, experiment 4 also demonstrated a radical reduction in MIP selectivity when introducing acidified PBS (pH 4.7). This suggests that at lower acidified $\mathrm{pH}$ levels

273 there are negative effects associate with protein binding. Under the acidified conditions for

274 the PBS, Tris and succinate buffer systems ( $\mathrm{pH} 4.7,5.4$, and 2.9 respectively) $-\mathrm{NH}_{2}$ groups in 275 both $\mathrm{MIP}_{\text {polyAA }}(\mathrm{pKa}=7.9)$ and template $\mathrm{BHb}$ protein $(\mathrm{pI}=6.8)$ are protonated [22]. The protein and MIP itself are therefore able to undergo subtle conformational changes or molecular relaxation under such acidic conditions. This combination of charge repulsion

278 between cationic MIP and cationic protein as well as the ensuing structural changes in both

279 under acidic conditions may be contributing to the diminished selectivity of the MIP.

280 Interestingly, this is also supported by Uysal et al [21] who demonstrated at best a 2:1 selectivity ratio for BHb MIP:NIP when they prepared and utilised at $\mathrm{pH}$ 4.0.

\subsection{Tris buffer conditioning}


284 Notable for its ability to drastically improve MIP selectivity, Tris buffer (pH 7.4) conditioning was applied to polyAA, polyNHMA and polyNiPAm gels for the selective imprinting of bovine haemoglobin $(\mathrm{BHb})$, equine myoglobin $(\mathrm{EMb})$ and bovine catalyse (BCat). It should be noted that MIP preparation throughout this section remained constantly in MilliQ water. Fig. 2 illustrates a comparison between an EMb-MIP ${ }_{\text {polyAA }}$ under MilliQ water and Tris buffer conditions. It is observed that less non-specific protein is present within the MIPs, and a higher protein concentration is observed in the 'elute' supernatants. This suggests that a higher degree of specifically bound protein is attained with Tris buffer ( $\mathrm{pH} 7.4)$ conditions. The NIPs were also less capable of non-specifically binding BHb and a higher detection of protein in the Load phase was noticed. Furthermore, all protein could be accounted for; accumulation of the protein recovered in the different wash fractions mirrored the same amount of protein that was originally added to the polymers.

Table 4 illustrates the optimised and improved selectivities and demonstrates that the Tris buffer is clearly having a strong beneficial effect on the selective binding of protein to the MIP. Conformational stability of proteins have been known to increase if anionic buffers are used above the pI of the protein (and conversely, if cationic buffers are used below the pI) [20]. At optimum pI, proteins contain carboxyl and amide groups existing as $-\mathrm{NH}_{3}{ }^{+}$and -

$\mathrm{COO}^{-}$. Above their $\mathrm{pI}$ however, proteins become negatively charged and the groups exist as $\mathrm{NH}_{2}$ and $-\mathrm{COO}^{-}$, (see Table 4). This overall negative net charge induces more favourable and complementary hydrogen bonding interactions, resulting in increased specific as well as nonspecific binding. The Tris buffer (pKa of 8.1) with its three un-dissociated hydroxyl groups at pH 7.4 seems suitable for improving MIP selectivity by providing optimum imprinting for specific and reducing non-specific binding interactions. It is plausible that the Tris buffer is aiding in stabilising the native protein structure in the fine protein-selective architecture 
309 imprinted within the MIP. Moreover, MIP selectivities demonstrate a varying affinity for

310 different protein-polymer combinations. Interestingly, the selectivity is highest for BHb-

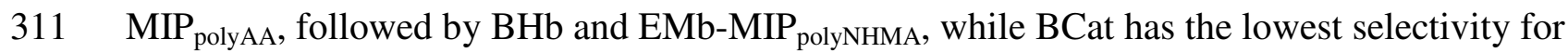

312 all MIPs. This provides some hints to the properties in terms of polymer and protein

313 interactions. It has been observed that with smaller size proteins a higher crosslinking density

314 is necessary [14]. EMb (17.5kDa) a quarter that of the size of BHb (64.5kDa) would therefore

315 require a MIP with a higher crosslinking density, otherwise protein cavities cannot be

316 optimised in order to reach full imprinting capacity. The opposite is also true for larger

317 proteins. For example, BCat $(250 \mathrm{kDa})$ is much larger and a much lower crosslinking density

318 is required. Control experiments have shown these densities to be optimum at $15 \%$ and $5 \%$

319 for EMb and BCat respectively. It should be noted that for consistency all hydrogels in this

320 paper were produced using a standard $10 \%$ crosslinking density. Therefore the low selectivity

321 exhibited by the MIPs towards BCat can be attributed to the latter, as only a few cavities will

322 be present due to a high crosslinking density. Moreover, MIP selectivity is highest for

323 polyAA, followed by polyNHMA and polyNiPAm gels. This can be attributed to the

324 hydrophobicity of the polymers; although polyNHMA is the most hydrophilic and

325 polyNiPAm the most hydrophobic, the results are suggesting that the neutral polyAA is

326 providing ideal binding affinities for this set of proteins within Tris buffer conditions.

328 To further expand on the Tris buffer effect ( $\mathrm{pH} 7.4)$, two series of gel conditioning

329 experiments (MilliQ water and Tris buffer $\mathrm{pH}$ 7.4) were conducted for a polyAA MIP.

330 Fig. 3 illustrates the positive effects of Tris introduction to the MIPs at both the conditioning

331 and loading phases. However, when MilliQ water is introduced into the Tris conditioning

332 series at the loading phase a staggering 0.8 selectivity was observed (Fig. 3, Table 5). This

333 was exhibited by both MIP and NIP and resulted in an unusually high amount of protein 
334 being irreversibly bound. Over $98 \%$ of the protein irreversibly bound to the NIP, while only

$33583 \%$ bound to the MIP (Fig. 3). The reason for this high degree of irreversible binding to the

336 NIP could be due to a change in conformation of the protein due to a change in $\mathrm{pH}$. Since

337 Tris is of $\mathrm{pH} 7.4$ and conditioning series have stabilised the gels, a MilliQ water load (pH 5.4)

338 would therefore induce conformational changes, and in turn alter the protein binding

339 characteristics. Another explanation could be that the Tris buffer is causing gel contraction

340 around the reloaded protein and trapping it within the polymer network [21-22]. Table 5

341 illustrates the experimental data collected and resulting MIP:NIP selectivity ratios $(\alpha)$

342 depending on the variant Tris buffer and MilliQ water conditioning series for MIP washing

343 and re-loading. It can be seen that the full Tris buffer conditioning series demonstrates its

344 superiority in terms of improving the imprinting efficiency resulting in a distinctively high

345 selectivity ratios $(\alpha)$ compared with the standard MilliQ water conditioning strategy.

\subsection{Biocompatibility study}

348 In order to assess their suitability in real biological samples, polyAA-BHb HydroMIPs were

349 investigated for their potential application for biological diagnostics using human plasma and serum matrices to assess for potential interferents that could affect template protein rebinding. Reload samples of diluted plasma or serum (1/10 or 1/100) in either MilliQ water or Tris buffer ( $\mathrm{pH} 7.4$ ) were spiked with a $3 \mathrm{mg} / \mathrm{ml}$ of BHb template. Figure 4 illustrates the $\%$ of $\mathrm{BHb}$ rebinding in terms of MIP imprinting efficiency after Tris buffer ( $\mathrm{pH}$ 7.4) preconditioning (Figure 4 a) and MilliQ water pre-conditioning (Figure 4 b) studies. The MIPs were tested for protein rebinding from the bio sample first in the medium that was used for pre-conditioning and then in the medium not used for pre-conditioning. The effects such of cross-loading in alternate media are also presented in Figs $4 \mathrm{a}$ and $\mathrm{b}$. The $\%$ of rebound $\mathrm{BHb}$ is calculated by subtracting the non-specific $\mathrm{BHb}$ bound in the NIP from the specific $\mathrm{BHb}$ 
bound to the MIP, divided by the initial reload concentration $(3 \mathrm{mg} / \mathrm{ml}) \times 100$. By subtraction

360 of protein binding in the NIP control from the MIP this correction allows us to effectively

361 demonstrate the absolute imprinting or rebind efficiency exhibited by our MIPs and eliminate any non-specific polymer matrix effect.

364 Varying percentages of BHb binding can be seen in Figure 4 depending on either the serum or plasma studies and also within the different dilution factors. This can most likely be attributed to the potential interference of the bio sample, i.e. globulins and clotting proteins [19]. It can be seen that a $1 / 10$ dilution of both serum and plasma has a hindrance on the rebinding efficiency of $\mathrm{BHb}$, and that the plasma has a higher hindrance effect then that of serum. However, it is still clear to see that Tris buffer conditioning followed by reloading in Tris diluted samples demonstrates its superiority in terms of MIP rebinding efficiency compared with the standard MilliQ water conditioning and reloading from water-diluted samples. This can also be seen, to an even greater extent, in the alternate media cross-loading studies. When MilliQ water is introduced into the Tris conditioning series at the loading phase (Figure $4 a$ ) less than $10 \%$ of protein was specifically bound. This was due to the unusually high amount of protein irreversibly binding in the NIP as well as MIP (resulting in this poor comparative rebinding efficiency) as previously discussed in the previous section.

378 The effective demonstration of our optimised buffer conditioning strategy in terms of MIP

379 rebinding efficiency using real biological human plasma and serum samples and the sensitivity, specificity and stability of protein MIPs would make attractive future rapid diagnostics and also potentially as a simple screening tool in the analytical community.

\section{Conclusions}


384 We have found that the selectivity of the MIP can be increased or decreased post-preparation depending on the buffer composition and conditions used and that it is the buffer conditioning of the MIP at the re-loading stage which primarily determines MIP selectivity. We were able to achieve new high MIP selectivities when preparing and reloading the MIP under Tris $(\mathrm{pH}$ 7.4) buffer conditions, compared with either PBS, water only or succinate buffer.

Acidification had a detrimental effect on MIP selectivity, likely due to protonation of both protein and MIP itself and the ensuing electrostatic repulsion, not allowing the protein to occupy MIP cavities. Our results also suggest a cautionary message to avoid loading in water only especially after conditioning the MIP or NIP with Tris buffer or PBS (pH7.4). By optimising buffer composition and $\mathrm{pH}$ conditions, we are achieving new record high selectivities for the MIP when compared with NIP. Biocompatibility studies have also demonstrated the effective use of our optimised buffer conditioning strategy in terms of MIP rebinding efficiency using real biological human plasma and serum samples. This study, in turn, has major implications in improving the selectivity of analytical tools for solid phase extraction and biosensors which are based on hydrogel-based molecularly imprinted polymers.

400

401

\section{Acknowledgements}

The authors wish to thank the EPSRC (EP/G014299/1) and NERC/ACTF (RSC) for supporting this work.

\section{References}

406 [1] M.E. Byrne, K. Park, N.A. Peppas, Adv Drug Deliver Rev 54 (2006) 149-161.

407 [2] K. Haupt, Chem Commun 34 (2003) 171-178. 

$1665-1671$

[4] J.M. González-Sáiz, M.A. Fernádez-Torroba, C. Pizarro, Eur Polym J 33 (1997) 475-485.

411 [5] X. Pang, G. Cheng, R. Li, S. Lu, Y. Zhang, Anal Chim Acta 550 (2005) 13-17.

412 [6] D.M. Hawkins, D. Stevenson, S.M. Reddy, Anal Chim Acta 542 (2005) 61-65.

413 [7] Y. Xia, T. Guo, M. Song, B. Zhang, B. Zhang, Biomacromolecules 6 (2005) 2601-2606.

414 [8] J.L. Liao, Y. Wang, S. Hjerten, Chromatographia 42 (1996) 259-262.

415 [9] S.H. Ou, M.C. Wu, T.C. Chou, C.C. Liu, Anal Chim Acta 504 (2004) 163-166.

416 [10] T.Y. Guo, Y.Q. Xia, G.J. Hao, M.D. Song, B.H. Zhang, Biomaterials 25 (2004) 59054175912.

418 [11] L.I. Andersson, J Chromatography B 739 (2000) 163-173.

419 [12] L.I. Andersson, Anal Chem 68 (1996) 111-117.

420 [13] D.E. Hansen, Biomaterials 28 (2007) 4178-4191.

421 [14] K. Mosbach, Molecular imprinting. Trends Biochem, Sci. 19 (1994) 9.

422 [15] T. Wei, S. Kaewtathip, K. Shing. J. Phys.Chem. C 113 (2009) 2053-2062.

423 [16] Q. Gai, F.Qu and Y. Zhang, Separation Science and Technology 45 (2010) 2394-2399.

424 [17] G. Wulff, Angewandte Chemie Int. Ed. Engl. 341 (995) 1812.

425

[18] Y.D. Livney, O.Ramon, E. Kesselman, U. Cogan, S. Mizrahi and Y. Cohen, Journal of

Polymer Science: Part B: Polymer Physics, 39 (2001) 2740-2750.

427 [19] S.M. Reddy, D.M. Hawkins, Q.T. Phan, D. Stevenson, K. Warriner, Sensors Actuators

428 B: Chem., 176 (2013) 190-197.

429 [20] J.N. Patton, A.F. Palmer, Langmuir 22 (2006) 2212-2221.

430 [21] A. Uysal, G. Demirel, E. Turan, T. Caykara, Anal Chim Acta 625 (2008) 110-115.

431 [22] T. Takeuchi, D. Goto and H. Shinmori, Analyst 132 (2007) 101-103. 
432 [23] N.W. Turner, C.W. Jeans, K.R. Brain, C.J. Allender, V. Hlady and D.W. Britt, 433 Biotechnol Prog 22 (2006) 1474. 